\title{
Right of the senior next of kin in possible medical error/negligence: case scenario, a death due to strangulated incisional hernia
}

\begin{abstract}
The deceased was a 60 year old female with a recently diagnosed right ovarian cyst and an incisional hernia. She complained of abdominal pain for a period of one and a half month duration. Abdominal pain for two weeks was her fist presenting complaint and she found her way to hospital at the end of the second week where she was diagnosed to have the above two conditions. During the next one month period she had been admitted three times to the same ward for the same complaint. Partially reducible incisional hernia with a small amount of free fluid in the right iliac fosse was the only significant finding of her last admission. On the second day following the last discharge she had vomited few times and became unresponsive to be pronounced dead at the Out Patient Department (OPD). The post mortem examination revealed strangulated hernia with small bowel gangrene. Strangulation of incisional hernia is a surgically correctable condition which contributes towards preventing a sudden unexpected death. Death of a patient despite of repeated admissions for the same illness without proper and timely medical intervention is an incident to be discussed. A patient seeks medical advice in order to achieve a relief and a possible cure. A patient relies on doctors' decisions for their life. On the other hand it is a right of a patient to obtain maximum possible care with the available facilities at a given setting. The case under discussion critically scrutinizes such issues within the parameters of an avoidable death. Forensic Pathologists not uncommonly encounter deaths that could have been prevented had the ideal intervention been done at the correct level and correct time. Even when the death is resultant upon an overt case of medical malpractice, most of the time no further discussion is raised unless the next of kin files a case of medical negligence. Retrospective clinico-pathological correlations and attempts of minimizing such further mishaps are rarely taken up spontaneously unless statutory obligations such as "mandatory reviews" are imposed. The post mortem examination is the final conclusive scientific measure in ascertaining the cause of death in sudden and unexpected deaths. An effective communication between the clinician and the Forensic Pathologist is pertinent in such a context to correlate the clinical and post mortem findings. This necessity is mandatory when medical negligence is alleged as in this case. The three hospital admissions with same symptoms within one month's time made the next of kin to assume a probable medical negligence. A medical error and medical negligence are two different entities. Medical negligence is a condition where many criteria including the duty of care of a doctor has to be proved by the prosecution. The authors as Forensic Pathologists would like to recommend that it is mandatory to have sound scrutiny of certain surgical deaths with "no-fault approach" similar to the process adopted with regard to maternal deaths in order to prevent recurrences of mishaps in future.
\end{abstract}

Keywords: bowel gangrene, incisional hernia, medical negligence, no-fault concept
Volume 6 Issue 6 - 2018

\author{
Ariyarathna HTDW, Hulathduwa SR \\ Senior Lecturer, Department of Forensic Medicine, University of \\ Sri Jayewardenepura, Nugegoda, Sri Lanka
}

\author{
Correspondence: Ariyarathna HTDW, Senior Lecturer, \\ Department of Forensic Medicine, University of Sri \\ Jayewardenepura, Nugegoda, Sri Lanka, \\ Email ariyaretna@sjp.ac.lk
} Received: September 28, 2018 | Published: December 04,
2018 
the underlying cause. Authors believe that most of the time, though the post mortem examination reveals many important aspects there is no effective way to convey that message to the clinicians. As a result similar to the existing maternal death investigation in Sri Lanka, it is highly beneficial to adopt a "no fault finding investigative process" for selected surgical deaths as well in order to prevent recurrences.

\section{Case report}

A sixty year old lady was diagnosed of having a right ovarian cyst and an incisional hernia upon admission to a territory care hospital following two weeks of abdominal pain. On the first admission severe tenderness had been noticed at the surgical scar (midline). The hernia was partially reducible and the facial deficit had been identified. No features of intestinal obstruction were identified and she had been discharged. Six days after the discharge she had developed the same abdominal pain and got admitted to the same hospital where she was managed again for two days with symptomatic treatments. In another three weeks' time she had developed lower abdominal pain again with constipation. She happened to get admitted to the same ward. Basic management had been done with ultrasound scan. This revealed small amount of free fluid in left iliac fosse with slightly distended bowel loops (Figure 1) \& (Figure 2). With other basic blood investigations and $\mathrm{x}$-rays she was discharged after three days of inward management with the plan of reviewing after one month at the surgical clinic. Anyhow after two days, she had died at home with a few episodes of vomiting. An inquest was held and the postmortem examination was performed revealing a strangulated hernia at the site of the incisional hernia with small bowel gangrene and generalized peritonitis (Figure $3)$.

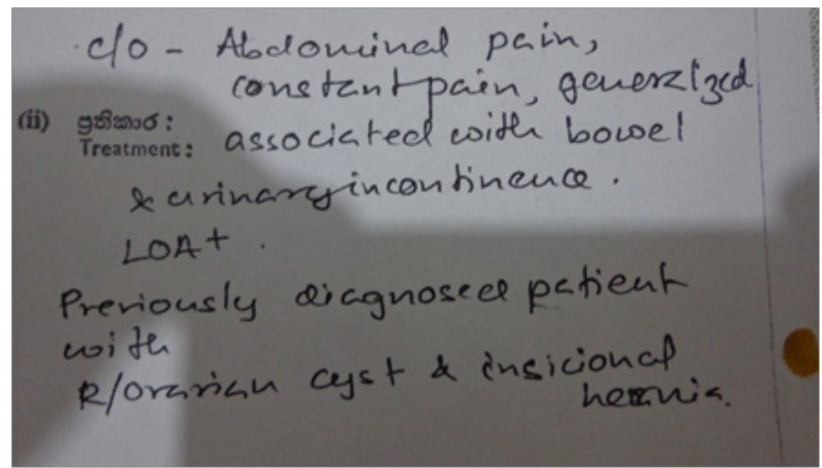

Figure I Patient's last diagnosis card (only the presenting complaint appears here).

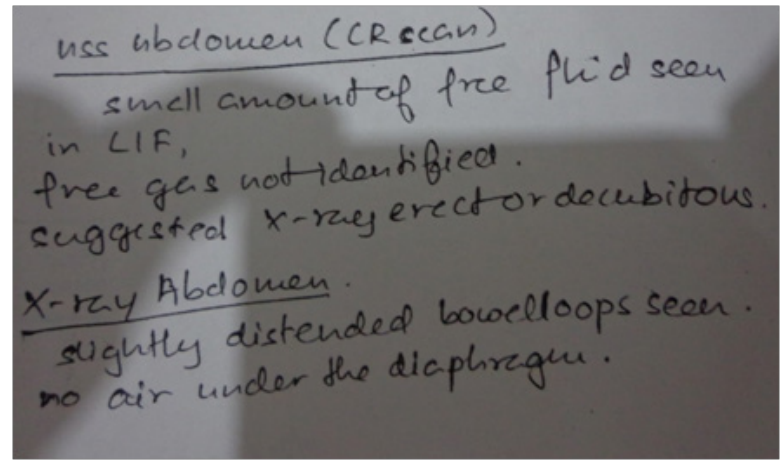

Figure 2 A part of investigative findings of her last admission.

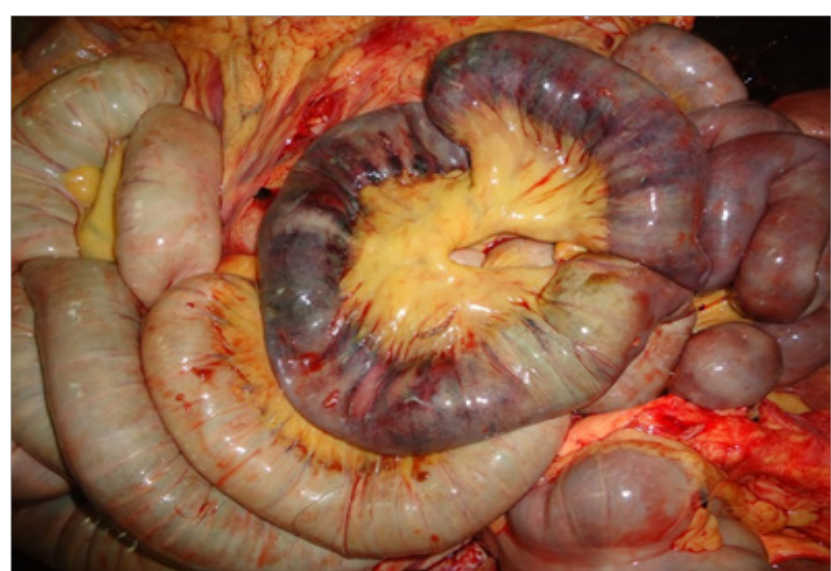

Figure 3 Note the gangrenous small bowel.

\section{Discussion}

Errors cannot be totally eliminated but can be minimized with many positive approaches. One such approach is to go for a court case. "No fault finding" inquiry/review is another way to protect patient's rights as that will help to scrutinize whether, when and how something has gone wrong and if so how to rectify the issues in future. Medical error and medical negligence are not synonymous. Medical error is a broader term which encompasses a wide array of errors that cause injury with variable severity. But when it comes to the medical negligence it deals with the preventable errors where there is a duty by the doctor. ${ }^{4}$ Strangulated hernia is a state of medical emergency and most of the complications are believed to be because of the lack of prompt treatment. ${ }^{5}$ Medical error has been identified as the third leading cause of death in the US and it is behind only to heart disease and cancer. ${ }^{6}$ According to the medical certificate of cause of death it must be mentioned that the chain of events that led to death of a particular person. So in an exercise of formulation of the cause of death, if it has to be written as medical error as a link that led to the death, what would be the outcome? The outcome would not be very pleasant, though the authors firmly believe that if there is a strong connection of medical error with the death, it has to be mentioned in the standard cause of death for certain pertinent reasons such as the principle of being totally truthful in medical practice, for statical purposes and as a justice to the deceased. In the ICD classification "medical error" has been recognized to some extent and it should be appreciated. ${ }^{7,8}$ As in the case under discussion, certain occasions the post mortem examination is the only remaining avenue to investigate into the cause of death. In such situations there are a lot of learning points that should be conveyed to the clinician treating doctors via Forensic Pathologists. But most of the time such discussions rarely happen in our country as it may be offensive for certain parties involved with the managing process. Maternal deaths are discussed in detail at different levels, institutional, regional levels and it is a very positive way forward in the Sri Lankan health system. (Maternal Death Surveillance \& Response System) Similarly in unexpected surgical deaths also such a process may be a timely need at least for a selected number of cases with the concept of "No-fault finding". In the case under discussion, there had been three hospital admissions almost within one months' time, and finally the patient died after two days of the discharge with the small bowel gangrene and peritonitis due to strangulated incisional hernia. There are many approaches 
in the surgical management of hernias. Watchful waiting is one of the accepted procedures but when there is minimal symptoms. ${ }^{9}$ In this case there had been recurrent admissions with certain type of symptoms where operative repair could have been a better approach according to the authors view as it largely may help to prevent the final undesirable outcome with death. The authors believe that it needs extensive scrutiny to find out the real cause for her incarcerated or strangulated hernia as at times it may be the fault of the patient thought they express the contrary that the patient died because of the medical error. Anyhow strangulated hernia can be considered as a surgically correctable situation in order to prevent sudden unexpected death. Death cannot be reversed. Diseases are also unavoidable. But there is a fair fraction of deaths that can be avoidable. As a result, it is the right of a patient to lead a quality life with best possible health care under the hands of doctors.

\section{Conclusion}

Making the correct diagnosis is one of the prime duties of a doctor If a prudent doctor fails to deliver reasonable degree of care to patient and further causes harm that may amount to medical negligence. If the desired medical management was implemented at the correct time prevention of death in certain occasions is highly probable. A retrospective study about such case scenarios is beneficial at all costs as it may prevent recurrences. The impact provided by a Forensic Pathologist in such an endeavor is essential as many unforeseen pathological states can be surfaced with the post mortem and other ancillary and special investigations.

\section{Acknowledgments}

None

\section{Conflicts of interest}

The author declares that there is no conflicts of interest.

\section{References}

1. Wallis K. Learning From No-Fault Treatment Injury Claims to Improve the Safety of Older Patients. The Annals of Family Medicine. 2015;13(5):472-474

2. Universal Declaration of Human Rights, United Nations. 1948.

3. Arianna B. 2017 update of the WSES guidelines for emergency repair of complicated abdominal wall hernias. World J Emerg Surg. 2017;12(37).

4. What's the Difference between Medical Errors, Mistakes and Malpractice?

5. Gilman, Bedigian. Strangulated hernia: Symptoms, treatment, and causes Medical News Today. 2018.

6. Medical error - the third leading cause of death in the US. BMJ. 2016.

7. Singh \& associates. Mondaq Connecting Knowledge and People. 2018.

8. Rosemary Roberts. Report of ICD-11 Revision Review. Consultancy Interim Assessment of 11th ICD Revision. 2015.

9. Klingensmith E. The Washington Manual of Surgery (Lippincott Manual Series). 6th edition. LWW, 2018. 